\title{
EUV and X-Ray Solar Flares Observed on-board the "Coronas-F" Satellite
}

\author{
A. A. Nusinov, T. V. Kazachevskaya, V. V. Katyushina
}

Institute of Applied Geophysics, Russian Hydrometeorological Service, Moscow email:geophys@hydromet.ru

\section{EUV instrumentation and measurements onboard CORONAS-F}

The CORONAS-F satellite has been launched on 31 July 2001 (Oraevsky et al. 2002). To conduct measurements of the intensity of solar radiation in the EUV spectral region near the hydrogen line $L_{\alpha}(\lambda=121.6 \mathrm{~nm})$ the spectrophotometer VUSS was installed onboard CORONAS-F. A vacuum photodiode was used as EUV detector in VUSS with the $C u J$ cathode and $M g F_{2}$ entrance window.

During a period of solar activity maximum a lot of flares were observed. The first observational results on EUV fluxes during these flares and their comparison with GOES 0.1-0.8 nm fluxes are presented below. As an example let us first consider the Solar flare of $X 5.3$ class (the optical class 3B), which took place on August 25, 2001, and was one of the most intensive flares registered by VUSS instrument. Fig.1 gives the VUSS measurements of this flare in the $L_{\alpha}$ line.

The figure also presents the temporal X-ray changes within the $0.1-0.8 \mathrm{~nm}$ wave-range observed on - board the GOES- 8 satellite. On Fig.1 a dip in the line corresponds to an entry of the satellite into a zone of twilight. A break was caused by a loss of information between communication sessions. It is evident that moments of maximum fluxes differ: an X-ray achieves maximum $\sim 13$ minutes later than EUV-emission. A scale of variations is also different: EUV emission changes by $\sim 2.7 \%$, while X-ray emission changes by the dozens of times. It is also important that duration of the flare in EUV region (about several seconds) is considerably shorter than in X-ray region. These characteristics are typical of the most flares observed.

A maximum of the most flares observed in EUV region occurs before an appearance of an X-ray maximum; EUV-emission leads X-ray emission by 2-13 minutes. Figure $2 a$ presents a dependence of time difference $\delta T$ (minutes) between flare maxima observed in EUV and X-ray regions, on emission intensity in 0.1-0.8 nm wave-range (GOES-8).

Fig. $2 a$ shows that $\delta T$ is increasing with an increase of a flare power. The tendency is evident though a spread in data is rather great.

One of the main characteristics is a power emitted in the maximum of a flare. Figure $2 b$ presents data on an energy of the flares in $L_{\alpha}$ depending on the 0.1-0.8 nm X-ray energy during a flare maximum. An unperturbed value of intensity in $L_{\alpha}$ line was taken from the paper (Nusinov 2004). On Fig.2b one can see relation between amplitudes of Solar flares in X-ray $(0.1-0.8 \mathrm{~nm})$ and EUV $(\sim 120 \mathrm{~nm})$ ranges. The points observed are well approximated by a straight line (it is shown on the Fig. $2 b$ ), i.e. there is a power law dependence between flare fluxes within the range of two orders of magnitude of X-ray changes. The same relation was obtained earlier by Kazachevskaya et al. 1996 and was also found for soft X-rays by Nusinov \& Chulankin 1997. 


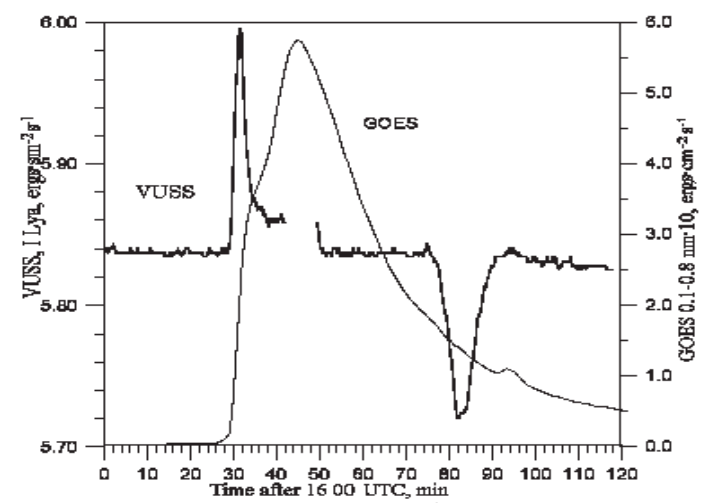

Figure 1. The flare of August 25, 2001: changes of VUSS and GOES(0.1-0.8 nm)
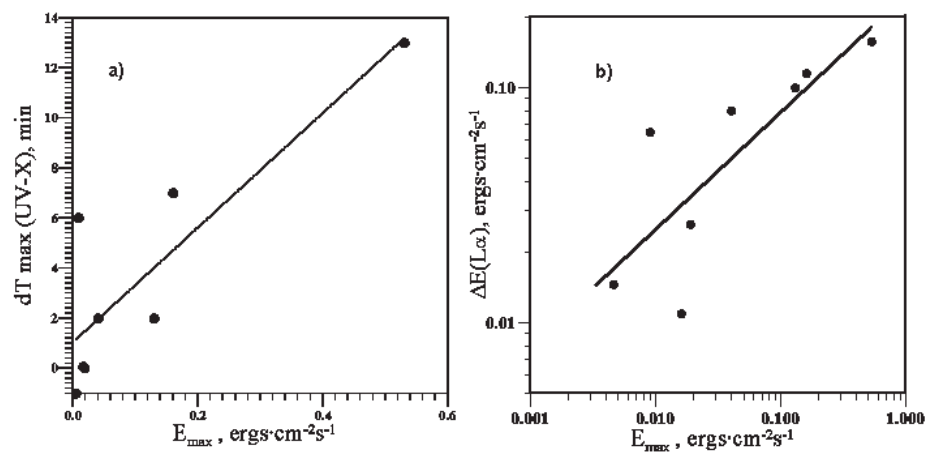

Figure 2. Dependence of $a$. time delay and $b$. EUV energy on X-ray flare energy.

\section{Conclusion}

1. Relative values of flares amplitudes in the band of $\sim 120 \mathrm{~nm}$ were found. It was confirmed that during even the most intensive flares an increase of emission in this band did not exceed several percents.

2. There is a power law correlation between soft X-ray and EUV emission. It allows to estimate EUV flux changes in the periods of flares on the basis of the X-ray patrol data.

3. Study of the EUV Solar flux variations in different wave-ranges revealed that at the beginning of a flare and during its maximum phase EUV emission systematically led soft X-ray emission by 2-5 minutes. To all appearance there is a possibility that a flare development takes place not in the corona's loops but in the upper layers of the Solar atmosphere.

\section{Acknowledgements}

The work was supported by the RFBR (project No. 03-02-17109).

\section{References}

Kazachevskaya T.V., Bruevich E.A. \& Ivanov-Kholodny G.S. 1986 Solnechnye Dannye 3, 68-72. Nusinov A.A. \& Chulankin D.I. 1997 Geomagnetism and Aeronomy 37, 14-23.

Nusinov A.A. 2004 Geomagnetism and Aeronomy 34, in press.

Oraevsky V.N., Sobelman I.I, Kuznetsov V.D. \& Zhitnik I.A. 2002 UFN 172, 949-959. 